\title{
Impressão 3D e Tecnologia Assistiva: um estudo de análise da produção científica nos últimos dez anos
}

\section{D Printing and Assistive Technology: a study of analysis of the scientific production in the last ten years}

\author{
Ana Lya Moya Ferrari ${ }^{1}$ \\ Aline Darc Piculo dos Santos ${ }^{2}$ \\ Thaísa Mara Cedano Godoy Patricio de Souza ${ }^{3}$ \\ Fausto Orsi Medola
}




\section{Resumo}

A impressão 3D é utilizada em diversas áreas, entretanto, pouco tem sido pesquisado com relação à sua utilização no desenvolvimento de Tecnologia Assistiva (TA). Este artigo apresenta uma análise dos trabalhos científicos publicados entre 2007 e 2018 relacionados a essa temática. $\mathrm{O}$ estudo avaliou as categorias de TA que mais utilizam impressão 3D, materiais empregados e países que mais investem no tema. Os resultados sugerem que a utilização destas tecnologias na fabricação de TAs é recente. Observou-se também que a impressão 3D é majoritariamente empregada na produção de órteses e próteses e os países desenvolvidos são os que mais investem nesta temática.

Palavras-chave: Tecnologia Assistiva; Impressão 3D; Prototipagem Rápida.

\section{Abstract}

The 3D printing is used in many areas; however, little has been researched regarding its use in the development of Assistive Technology (AT). This paper presents an analysis of scientific papers published between 2007 and 2018 related to this issue. The study evaluated the categories of TA that most use 3D printing, materials employed and countries that invest the most in the theme. The results suggest that the use of these technologies in the manufacture of TAs is recent. It was also observed that 3D printing is mostly used in the production of orthoses and prostheses and the developed countries are the ones that invest the most in this theme.

Key-words: Assistive Technology; 3D printing; Rapid Prototyping.

${ }^{1}$ Mestranda em Design, UNESP (analya_mf@hotmail.com) ${ }^{2}$ Mestre em Design, UNESP (alinedarcps@gmail.com)

${ }^{3}$ Mestranda em Design, UNESP (thaisapatricio@gmail.com)

${ }^{4}$ Doutor em Ciências, UNESP (fausto.medola@faac.unesp.br) 


\section{INTRODUÇÃO}

As tecnologias de impressão 3D estão cada vez mais ganhando destaque devido, principalmente, à divulgação pela mídia, desenvolvimento de novas técnicas e materiais, aumento das tecnologias de baixo custo e softwares de código aberto inseridos no mercado. Além disso, atualmente, as impressoras 3D e o conhecimento necessário para fabricar objetos com essas tecnologias estão se tornando cada vez mais acessíveis para a população (OSTUZZI et al., 2015).

Os processos de manufatura aditiva (MA), na qual insere-se a impressão 3D, consistem no método de produção por deposição de material em camadas, segundo especificações provenientes de modelos desenvolvidos virtualmente através de softwares CAD (Computer-Aided Design). Através destes métodos é possível produzir peças anteriormente inviáveis em pequena escala ou até mesmo impossíveis de se confeccionar (OSTUZZI et al., 2015; DODZIUK, 2016). Tais aspectos oferecem aos designers maior liberdade no desenvolvimento de objetos, gerando peças com diferentes formas, durabilidade e alta resistência, além de produtos personalizados que atendam às necessidades específicas dos usuários. Além disso, a impressão 3D também pode ser uma alternativa mais econômica em detrimento aos métodos tradicionais de produção, especialmente no que se refere à criação de produtos únicos e personalizados como é necessário, muitas vezes, no campo da saúde.

Os materiais empregados na impressão 3D são diversos, englobando plásticos como ácido politáctico (PLA), polipropileno (PP), acrilonitrilo butadieno estireno (ABS), nylon; metais, como o titânio e aço inoxidável, fibras de carbono, borracha e vidro, além de materiais biocompatíveis que são utilizados para a produção de tecidos, células ou implantes (TELFER et al., 2012; MARKSTEDT et al., 2015; DODZIUK, 2016). A diversidade de técnicas de impressão e materiais utilizados permitem o emprego dessas tecnologias em diferentes áreas, possibilitando desde a produção de peças industriais até dispositivos para a área da saúde.

Com a sua crescente popularização e os constantes avanços tecnológicos, o uso da impressão 3D se tornou foco de diversos estudos que abordam sua aplicação nas mais variadas áreas, com grande destaque para a fabricação de dispositivos auditivos e para aplicações odontológicas, no desenvolvimento de próteses e implantes. Segundo Dodziuk (2016), a utilização da impressão 3D na área da saúde pode ser dividida nas seguintes categorias gerais: criação de próteses, implantes e modelos anatômicos; fabricação de tecidos e órgãos e fabricação de instrumentos cirúrgicos e dispositivos médicos. Outro campo de aplicação da impressão 3D é a área da medicina física, com a produção de órteses e demais dispositivos utilizados na reabilitação e melhoria funcional dos pacientes (MICLAUS; REPANOVICl; ROMAN, 2017).

Estudos recentes apontam vantagens na utilização da impressão 3D na área da medicina (RENGIER et al., 2010; MARTELLI et al., 2016; KAYE et al., 2016). Tais estudos demonstram que as próteses e órteses são o campo no qual mais se utiliza a impressão 3D (RENGIER et al., 2010; MARTELLI et al., 2016; KAYE et al., 2016). Para Telfer et al. (2012), a prototipagem rápida é importante pois possibilita uma nova abordagem da forma das órteses, tanto na fabricação de dispositivos anatomicamente ajustados, 
quanto no desenvolvido de peças com novas morfologias.

A educação especial e a confecção de dispositivos assistivos para crianças são outros campos da Tecnologia Assistiva que também podem se beneficiar com as impressões 3D. Nesse caso, as tecnologias de baixo custo são interessantes, uma vez que crianças perdem seus dispositivos de Tecnologia Assistiva em um curto espaço de tempo, devido ao crescimento ou por não se adaptarem a eles (BUEHLER et al., 2016). Apesar de seu potencial de uso, não foram encontrados estudos de revisão que discutem de que forma as tecnologias de impressão 3D vêm sendo utilizadas no projeto das diversas categorias de dispositivos de TAs.

Considerando as diversas possibilidades de contribuição da prototipagem rápida no desenvolvimento de produtos de TA, o presente trabalho tem como objetivo verificar o panorama geral da produção científica relacionando os temas Tecnologia Assistiva e Impressão 3D, a fim de compreender como estas tecnologias estão sendo utilizadas na produção de TAs. Com isso, será possível compreender melhor seu impacto para os dispositivos assistivos, bem como propor um direcionamento para futuros estudos.

\section{METODOLOGIA}

Este estudo de revisão de literatura é estruturado em duas etapas. Na primeira, realizou-se uma busca de artigos científicos na base de dados Web of Science, no período de dezembro de 2016 a abril de 2018, sobre a temática impressão 3D e Tecnologia Assistiva, utilizando os seguintes pares descritores: "3D printing; Assistive Device"; "3D printing; Assistive Technology"; "3D printing; Orthosis"; "Communication; Assistive Technology; Rapid prototyping"; "Disability; 3D printing; Assistive"; "Visual Impairment; 3D printing"; "3D printing; Prosthesis" e "3D printing; Amputees". Para facilitar a filtragem dos termos descritores, foi utilizado o operador lógico "and".

Como critérios de inclusão, foram analisados apenas artigos publicados em periódicos científicos durante o período entre 2007 e abril de 2018, compreendendo os últimos dez anos, para que os trabalhos analisados pudessem ser recentes, além de verificar quando começam a surgir as pesquisas sobre esse tema e se elas estão aumentando.

Todos os estudos deveriam abordar concomitantemente a utilização da impressão 3D voltada ao desenvolvimento de Tecnologias Assistivas, pois embora as buscas realizadas tenham utilizado termos específicos relacionados à TA como palavras chaves, algumas pesquisas poderiam abordar outros tipos de tecnologia que não se encaixam nos objetivos deste trabalho. Com isso, qualquer trabalho que não atendeu a esses critérios, como artigos publicados em eventos, trabalhos publicados anteriormente ao ano de 2007, ou trabalhos que abordavam apenas um dos temas, como impressão 3D ou Tecnologia Assistiva, foram descartados.

\section{RESULTADOS}

Foram encontrados 227 artigos, dos quais 136 se encaixavam nos critérios de inclusão. O par descritor que retornou o maior número de resultados foi " $3 D$ printing 
+ prosthesis", com 136 resultados. Apesar do elevado número de artigos, a maioria dos resultados referiam-se às endopróteses, que não são consideradas dispositivos de Tecnologia Assistiva. Realizou-se a leitura dos resumos dos artigos para verificação de enquadramento no tema, sendo selecionados 25 que atendiam à temática da pesquisa, conforme descrito na Tabela 1. Os artigos selecionados foram aqueles nos quais a impressão 3D foi utilizada no desenvolvimento de dispositivos ou parte de dispositivos de TA, ou que ao menos discutissem a utilização desse tipo de tecnologia na produção desses produtos. Após a seleção dos artigos, foi realizada uma leitura detalhada a fim de analisar o conteúdo dos estudos, a partir dos seguintes critérios: ano de publicação, área temática da revista na qual o artigo foi publicado, país no qual foi realizado o estudo, categoria de TA abordada, tipos de materiais e técnicas de impressão 3D utilizados. É interessante destacar que, em alguns estudos, os próprios pacientes participaram do design de seus dispositivos, opinando sobre modelo e usabilidade.

\begin{tabular}{|c|c|c|c|c|}
\hline & Título do trabalho & Local de Publicação & País & Ano \\
\hline 1 & $\begin{array}{c}\text { Embracing additive manufacture: } \\
\text { implications for foot and ankle } \\
\text { orthosis design }\end{array}$ & $\begin{array}{l}\text { BMC Musculoskeletal } \\
\text { Disorders }\end{array}$ & $\begin{array}{l}\text { Reino } \\
\text { Unido }\end{array}$ & 2012 \\
\hline 2 & $\begin{array}{c}\text { Dose-response effects of customized } \\
\text { foot orthoses on lower limb } \\
\text { kinematics and kinects in pronated } \\
\text { foot type }\end{array}$ & Journal of Biomechanics & $\begin{array}{l}\text { Reino } \\
\text { Unido }\end{array}$ & 2013 \\
\hline 3 & $\begin{array}{c}\text { Dose-response effects of customized } \\
\text { foot orthoses on lower limb muscle } \\
\text { activity and plantar pressures in } \\
\text { pronated foot type }\end{array}$ & Gait \& Posture & $\begin{array}{l}\text { Reino } \\
\text { Unido }\end{array}$ & 2013 \\
\hline 4 & $\begin{array}{c}\text { Improving Tactile Map Usability } \\
\text { through 3D Printing Techniques: } \\
\text { An Experiment with New Tactile } \\
\text { Symbols }\end{array}$ & The Cartographic Journal & Espanha & 2013 \\
\hline 5 & $\begin{array}{c}\text { Pilot study of the wrist orthosis } \\
\text { design process }\end{array}$ & Rapid Prototyping Journal & $\begin{array}{l}\text { República } \\
\text { Tcheca }\end{array}$ & 2014 \\
\hline 6 & $\begin{array}{c}\text { (+) TUO project: low cost 3D printers } \\
\text { as helpful tool for } \\
\text { small communities with rheumatic } \\
\text { diseases }\end{array}$ & Rapid Prototyping Journal & $\begin{array}{l}\text { Itália e } \\
\text { Bélgica }\end{array}$ & 2015 \\
\hline 7 & $\begin{array}{c}\text { Development and Evaluation of a } \\
\text { CAD/3DP Process for Transtibial } \\
\text { Socket Fabrication }\end{array}$ & $\begin{array}{l}\text { Biomedical Engineering: } \\
\text { Applications, Basis and } \\
\text { Communications }\end{array}$ & Taiwan & 2015 \\
\hline 8 & $\begin{array}{l}\text { Development and validation of a } \\
\text { 3D-printed interfacial stress sensor } \\
\quad \text { for prosthetic applications }\end{array}$ & $\begin{array}{c}\text { Medical Engineering \& } \\
\text { Physics }\end{array}$ & $\begin{array}{l}\text { Reino } \\
\text { Unido }\end{array}$ & 2015 \\
\hline 9 & $\begin{array}{c}\text { Fabricación Digital Directa para } \\
\text { Deportes y Aplicaciones Médicas: } \\
\text { Tres Casos Prácticos }\end{array}$ & Dyna Ingeneria e Industria & Espanha & 2015 \\
\hline 10 & $\begin{array}{l}\text { Smart Knee Brace Design with } \\
\text { Parallel Coupled Compliant Plate } \\
\text { (PCCP) Mechanism and Pennate } \\
\text { Elastic Band (PEB) Spring }\end{array}$ & $\begin{array}{c}\text { Journal of Mechanisms and } \\
\text { Robotics }\end{array}$ & $\begin{array}{l}\text { Estados } \\
\text { Unidos }\end{array}$ & 2015 \\
\hline
\end{tabular}




\begin{tabular}{|c|c|c|c|c|}
\hline 11 & $\begin{array}{c}\text { Development of novel 3D-printed } \\
\text { robotic prosthetic for transradial } \\
\text { amputees }\end{array}$ & $\begin{array}{l}\text { Prosthetics and Orthotics } \\
\text { International }\end{array}$ & $\begin{array}{l}\text { Estados } \\
\text { Unidos }\end{array}$ & 2016 \\
\hline 12 & $\begin{array}{c}\text { A Critical Analysis of a Hand Orthosis } \\
\text { Reverse Engineering and 3D Printing } \\
\text { Process }\end{array}$ & $\begin{array}{l}\text { Applied Bionics and } \\
\text { Biomechanics }\end{array}$ & Itália & 2016 \\
\hline 13 & $\begin{array}{c}\text { A Low-cost Open Source } \\
\text { 3D-Printable Dexterous } \\
\text { Anthropomorphic Robotic Hand with } \\
\text { a Parallel Spherical Joint Wrist for } \\
\text { Sign Languages Reproduction }\end{array}$ & $\begin{array}{l}\text { International Journal of } \\
\text { Advanced Robotic Systems }\end{array}$ & Itália & 2016 \\
\hline 14 & $\begin{array}{c}\text { Experimental studies of the quality of } \\
\text { embossed characters of the Braille } \\
\text { alphabet }\end{array}$ & $\begin{array}{l}\text { Bulletin of the Polish } \\
\text { Academy of Sciences } \\
\text { Technical Sciences }\end{array}$ & Polônia & 2016 \\
\hline 15 & $\begin{array}{l}\text { Investigating the Implications of 3D } \\
\text { Printing in Special Education }\end{array}$ & $\begin{array}{l}\text { ACM Transactions on } \\
\text { Accessible Computing }\end{array}$ & $\begin{array}{l}\text { Estados } \\
\text { Unidos }\end{array}$ & 2016 \\
\hline 16 & $\begin{array}{c}\text { Customized 3D printed ankle-foot } \\
\text { orthosis with adaptable carbon fibre } \\
\text { composite spring joint }\end{array}$ & Cogent Engineering & $\begin{array}{l}\text { Nova } \\
\text { Zelândia }\end{array}$ & 2016 \\
\hline 17 & $\begin{array}{c}\text { Design and Fabrication of a Six } \\
\text { Degree-of-Freedom Open Source } \\
\text { Hand }\end{array}$ & $\begin{array}{c}\text { IEEE Transactions On Neural } \\
\text { Systems And Rehabilitation } \\
\text { Engineering }\end{array}$ & $\begin{array}{l}\text { Estados } \\
\text { Unidos }\end{array}$ & 2016 \\
\hline 18 & $\begin{array}{c}\text { 'Limbitless Solutions': the Prosthetic } \\
\text { Arm, Iron Man and the Science } \\
\text { Fiction of Technoscience }\end{array}$ & Medical Humanities & $\begin{array}{l}\text { Reino } \\
\text { Unido }\end{array}$ & 2016 \\
\hline 19 & $\begin{array}{l}\text { Anisotropic flexible mechanical } \\
\text { design of prosthetic socket liner }\end{array}$ & $\begin{array}{c}\text { International Journal of } \\
\text { Applied Electromagnetics } \\
\text { and Mechanics }\end{array}$ & China & 2016 \\
\hline 20 & $\begin{array}{c}\text { Additive Manufacturing to Advance } \\
\text { Functional Design: An Application in } \\
\text { the Medical Field }\end{array}$ & $\begin{array}{c}\text { Journal of Computing and } \\
\text { Information Science in } \\
\text { Engineering }\end{array}$ & Itália & 2017 \\
\hline 21 & $\begin{array}{c}\text { Optimal kinematic synthesis and } \\
\text { fabrication of a one-DoF prosthetic } \\
\text { finger }\end{array}$ & $\begin{array}{l}\text { Journal of the Brazilian } \\
\text { Society of Mechanical } \\
\text { Sciences and Engineering }\end{array}$ & Irã & 2017 \\
\hline 22 & $\begin{array}{c}\text { Design and optimization of } \\
\text { prosthetic foot by using polylactic } \\
\text { acid 3D printing }\end{array}$ & $\begin{array}{l}\text { Journal of Mechanical } \\
\text { Science and Technology }\end{array}$ & $\begin{array}{l}\text { Coréia } \\
\text { do Sul }\end{array}$ & 2017 \\
\hline 23 & $\begin{array}{c}\text { Map Learning with a 3D Printed } \\
\text { Interactive Small-Scale Model: } \\
\text { Improvement of Space and Text } \\
\text { Memorization in Visually Impaired } \\
\text { Students }\end{array}$ & $\begin{array}{l}\text { Journal Frontiers in } \\
\text { Psychology }\end{array}$ & $\begin{array}{l}\text { França e } \\
\text { Singapura }\end{array}$ & 2017 \\
\hline 24 & $\begin{array}{c}\text { Design of a 3D-printed, open-source } \\
\text { wrist-driven orthosis for individuals } \\
\text { with spinal cord injury }\end{array}$ & Plos One & $\begin{array}{l}\text { Estados } \\
\text { Unidos }\end{array}$ & 2018 \\
\hline 25 & $\begin{array}{c}\text { Quantitative functional evaluation } \\
\text { of a 3D-printed silicone-embedded } \\
\text { prosthesis for partial hand } \\
\text { amputation: A case report }\end{array}$ & Journal of Hand Therapy & Chile & 2018 \\
\hline
\end{tabular}

Tabela 1 - Trabalhos selecionados que atenderam aos critérios de inclusão em ordem cronológica. Fonte: elaborado pelos autores (2018). 
Com relação ao ano de publicação, é possível observar, na Figura 1, que a maioria dos trabalhos foi publicada nos anos de 2015, 2016 e 2017, com 20\%, 36\% e 16\% das publicações respectivamente, demonstrando que o uso da impressão 3D na Tecnologia Assistiva é um tema de estudo recente e com abordagem crescente nos últimos anos. Neste gráfico observa-se, também, que os trabalhos abordando estes temas de forma conjunta começam a surgir a partir do ano de 2012, e que a partir de então a quantidade de publicações tem aumentado gradativamente.

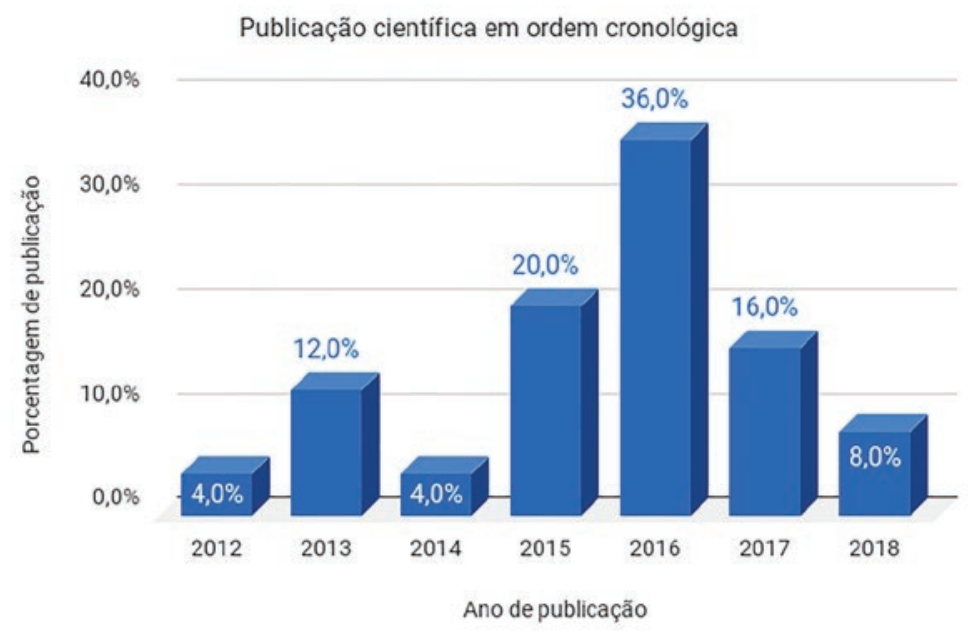

Figura 1 - Gráfico de análise da porcentagem de artigos publicados por ano. Fonte: elaboração dos autores (2018).

Quanto à categorização segundo o país de publicação (Figura 2), verifica-se que a maioria dos artigos foi publicada em países desenvolvidos, como Reino Unido e Estados Unidos com 5 publicações cada e Itália com 4 publicações. É importante ressaltar que alguns estudos foram realizados e publicados em parceria entre alguns países. Além disso, a maior parte dos artigos publicados concentram-se no continente europeu. No continente americano, Estados Unidos e Chile são os únicos países que apresentam publicações sobre a temática do estudo. Neste levantamento, não foi encontrado estudos publicados desenvolvidos no Brasil.

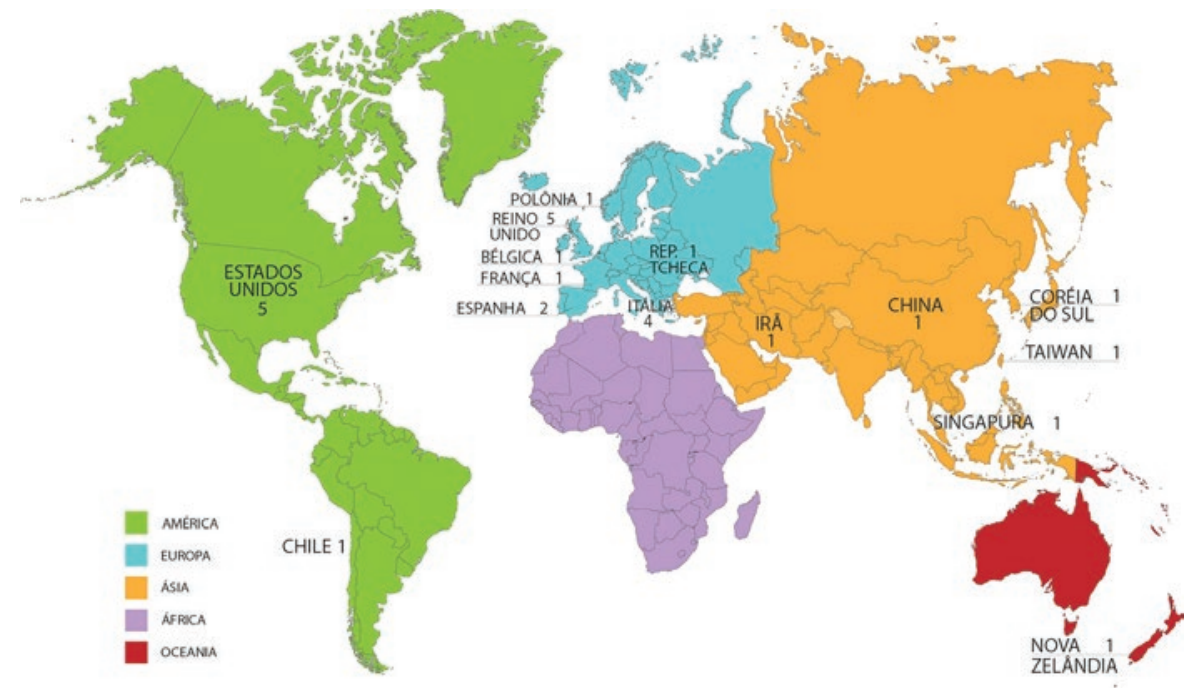

Figura 2 - Infográfico da quantidade de artigos publicados por país. Fonte: elaboração dos autores (2018). 
Quanto aos tipos de Tecnologia Assistiva (TA), observa-se, na Figura 3, uma diferença significativa entre o número de estudos relacionados às órteses e próteses em relação às demais TAs. A produção científica relacionada às órteses e próteses correspondem a $73,1 \%$ dos estudos encontrados, seguido pelos auxílios para pessoas cegas ou com baixa visão, porém com um número significativamente menor de publicações (11,5\%). Este mesmo fato foi observado em estudos anteriores de revisão bibliográfica que analisavam as aplicações da TA no campo da medicina (RENGIER, 2010; MARTELLI, 2016; KAYE, 2016). Neste caso, pode-se observar que os segmentos ortóticos e protéticos são bastante estudados, entretanto, quando se analisa o quadro geral de estudos relacionados à TA e prototipagem rápida, nota-se como os outros campos são carentes de pesquisas.

Apesar do grande número de estudos sobre dispositivos de auxílio locomotor como cadeiras de rodas e andadores, não foram encontrados, nesta revisão, publicações relacionadas à aplicação de impressão 3D na produção destes dispositivos.

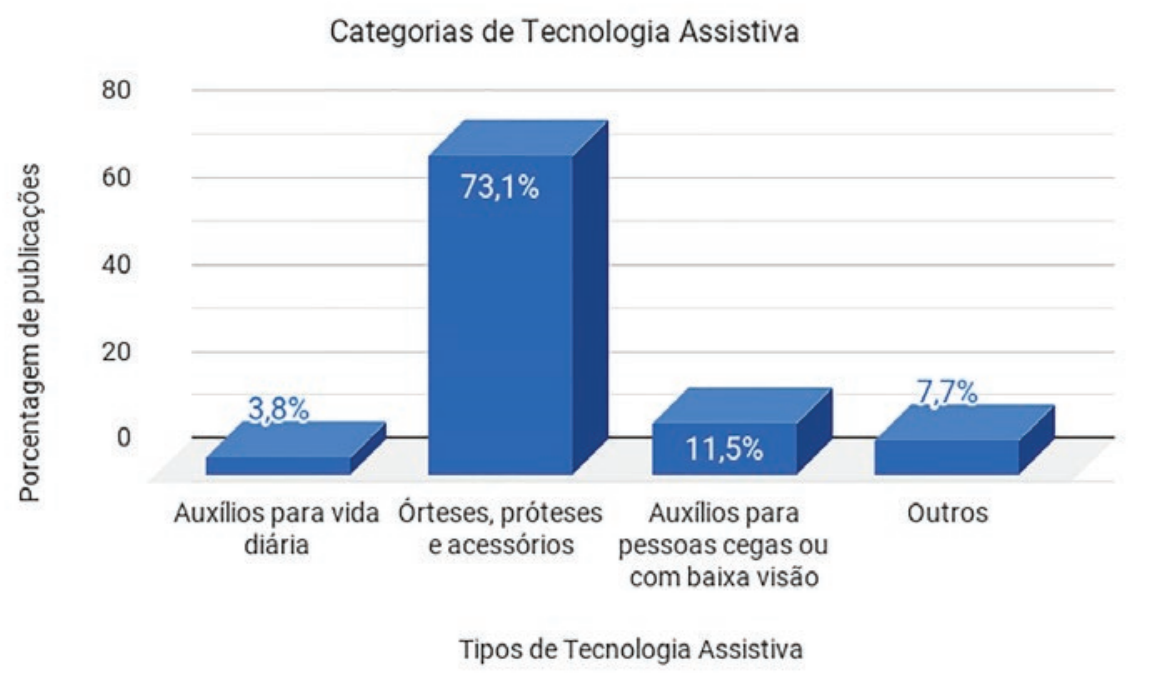

Figura 3 - Gráfico da porcentagem de artigos publicados por categorias de Tecnologia Assistiva. Fonte: elaboração dos autores (2018).

Além disso, observa-se que, na Figura 4, embora a maioria dos resultados encontrados seja relacionado às órteses de membros inferiores (MMII), a diferença em relação aos mesmos dispositivos para membros superiores (MMSS) é pequena, o que indica que os estudos sobre próteses e órteses estão sendo realizados em todos os segmentos. 


\section{Categorização quanto aos tipos de órtese, prótese e acessórios}

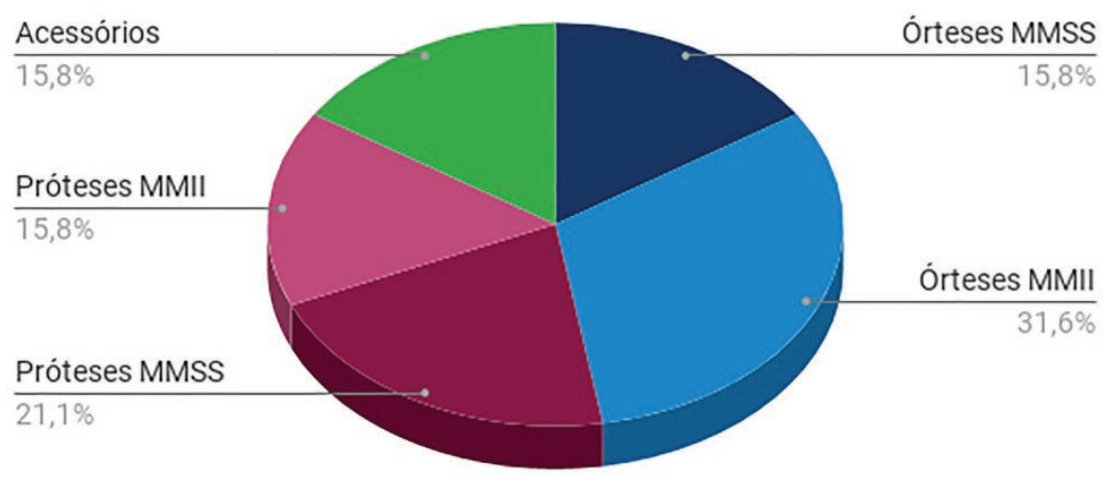

Figura 4 - Gráfico da quantidade de estudos encontrados por tipos de órteses, próteses e acessórios. Fonte: elaboração dos autores (2018).

Dos artigos selecionados, 22 estudos envolviam a impressão 3D do produto final ou de um protótipo; entretanto, somente 19 identificavam os materiais utilizados na produção. É possível observar que os dispositivos de TA foram fabricados predominantemente com polímeros, com maior destaque para ABS e PLA (Figura 5). Tais materiais apresentam significativa resistência térmica e ao impacto, características fundamentais tanto na impressão 3D quanto para a confecção de dispositivos de TA, além de apresentarem custo relativamente baixo. Além disso, alguns estudos também mencionam o uso de scanner, fundamental em ocasiões onde o produto final será projetado sob medida para os pacientes.

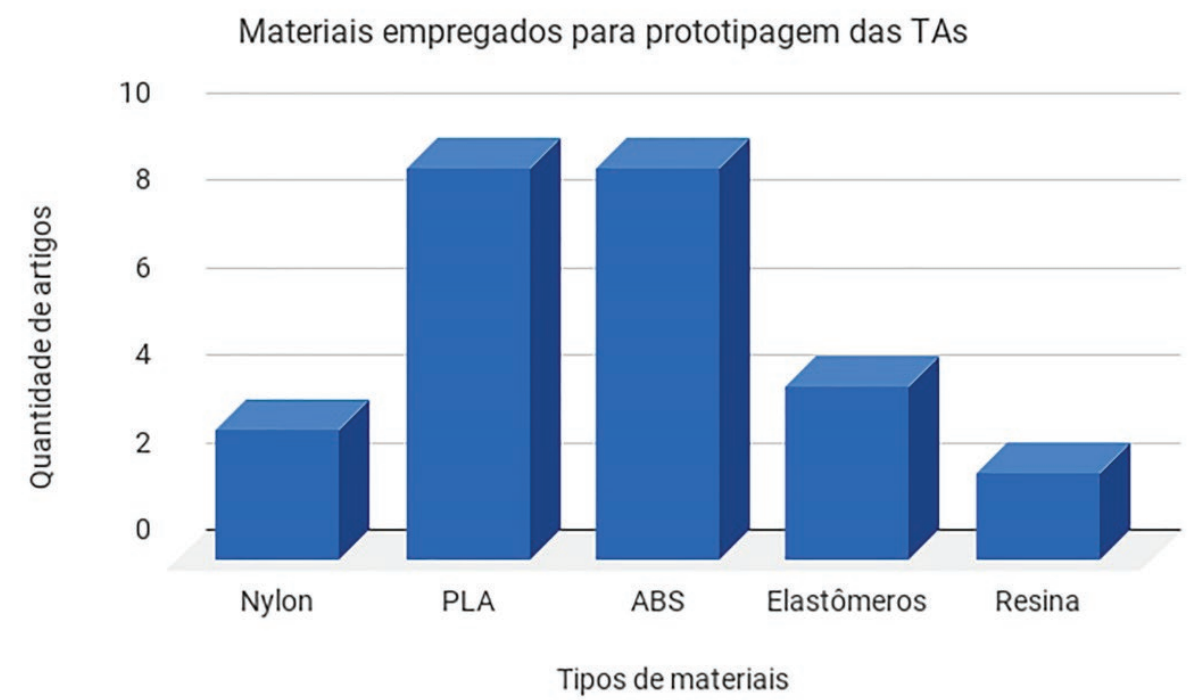

Figura 5 - Gráfico dos tipos de materiais mais utilizados na impressão 3D de dispositivos de TA. Fonte: elaboração dos autores (2018).

Quanto ao tipo de Tecnologia usada na Prototipagem Rápida (Figura 6), percebe-se que a maioria fez o uso da FDM (62\%) para fabricar seus produtos, número muito superior a segunda mais utilizada, a SLS (11\%). No total, seis tipos de tecnologia 
foram aplicadas nos trabalhos selecionados, sendo que três deles não informaram a técnica empregada. Além disso, um dos trabalhos selecionados apresenta o uso de duas técnicas no mesmo estudo.

Tecnologias de Impressão 3D

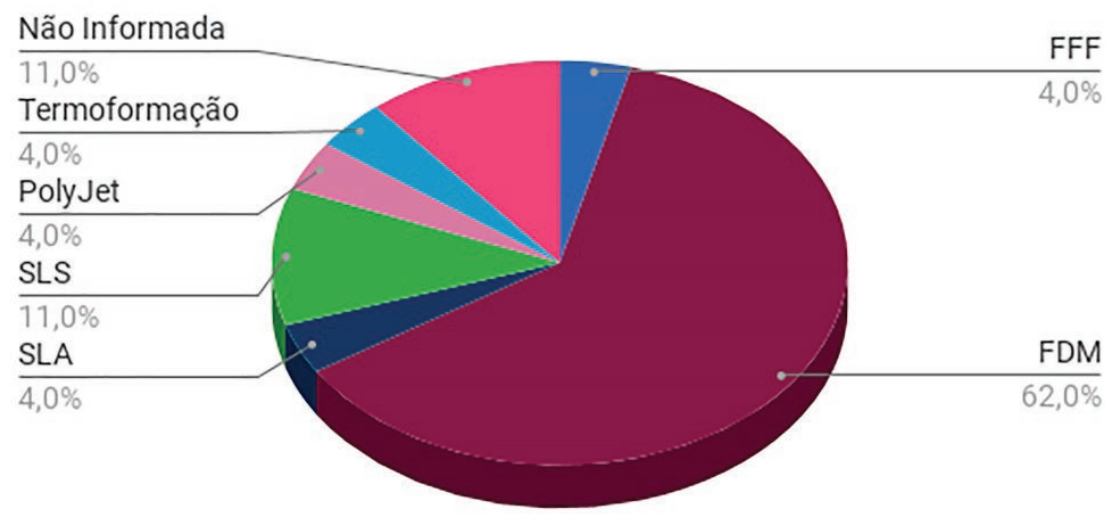

Figura 6 - Gráfico dos tipos de tecnologia utilizadas na impressão 3D de dispositivos de TA. Fonte: elaboração dos autores (2018).

O uso superior da técnica FDM (Fused Deposition Modeling) pode ser explicado pelo fato de utilizar como matéria um termoplástico, que permite produzir peças a partir do aquecimento e da extrusão deste através de um bico, produzindo as peças camada por camada, de baixo para cima, até que se construa a forma desejada, sendo assim uma técnica de custo acessível e que possibilita a criação de peças mais resistentes.

\section{CONSIDERAÇÕES FINAIS}

A crescente utilização das tecnologias de impressão 3D configura-se como uma grande aliada na área da saúde. A redução do tempo, o aumento das tecnologias de baixo custo e a possibilidade de personalização de objetos adequados aos usuários são as principais vantagens proporcionadas por tais tecnologias.

Este estudo realizou uma revisão sistemática dos trabalhos científicos publicados no período de 2007 a abril de 2018, a fim de avaliar a produção científica relacionando o uso da impressão 3D no planejamento e confecção de dispositivos de Tecnologia Assistiva. Foram encontrados 227 artigos, dos quais somente 25 se encaixaram nos critérios de inclusão, demonstrando que, embora a impressão 3D seja um tema atual e foco de muitas pesquisas, pouco ainda se tem estudado com foco na Tecnologia Assistiva.

Os resultados sugerem que a associação entre a Prototipagem Rápida e Tecnologia Assistiva é uma temática ainda recente, visto que os primeiros trabalhos encontrados foram publicados a partir do ano de 2012, não sendo encontrada nenhuma publicação anterior a este ano; com maior concentração de publicação entre os anos de 2015 e 2017, mostrando a sua progressiva utilização e aumento de pesquisas com foco na área. Este aumento pode ser relacionado aos avanços tecnológicos de prototipagem rápida que também crescem gradativamente. Além disso, verificou-se, 
também, o uso crescente da prototipagem rápida na área da saúde, principalmente na Ortodontia, cirurgias e implantes médicos.

No campo da Tecnologia Assistiva, entretanto, ainda são poucos os estudos que abordam a utilização da impressão 3D. As próteses e órteses são os dispositivos de TA que mais utilizam a impressão 3D, visto que foram os termos descritores com maior número de resultados encontrados. Com relação aos materiais, observou-se que os polímeros PLA e ABS foram os mais utilizados, possivelmente devido ao fato destes materiais serem utilizados nas técnicas de impressões por FDM (Fused Deposition Modeling), processo de custo relativamente mais baixo. Observou-se também que países desenvolvidos como Reino Unido, Estados Unidos e Itália são os que mais concentram a produção científica nos temas aqui abordados.

Deve-se ressaltar ainda, como limitações deste estudo, a escolha de descritores e do meio de busca utilizados para a pesquisa, que podem ter deixado de fora outras publicações sobre a temática.

De maneira geral, pode-se concluir que o uso da impressão 3D no campo da Tecnologia Assistiva é uma ferramenta muito vantajosa pois possibilita a individualização dos produtos fabricados, o que pode contribuir para a aceitação e satisfação do maior número de usuários, além de propiciar um produto com maior precisão e conforto. O presente trabalho demonstra a relevância da prototipagem 3D na fabricação de produtos para Tecnologia Assistiva, e a importância de se estudar novos materiais e meios de implantar progressivamente esse método, aumentando a acessibilidade para pacientes dependentes dessas tecnologias.

\section{REFERÊNCIAS}

BARCZYK, R.; CHOROMAŃSKA, D. J. Experimental studies of the quality of embossed characters of the Braille alphabet. The Journal of Polish Academy of Sciences. v. 64, n. 3, p. 607-614. 2016.

BARONIO, G.; HARRAN, S.; SIGNORONI, A. A Critical Analysis of a Hand Orthosis Reverse Engineering and 3D Printing Process. Applied Bionics and Biomechanics. v. 2016. p. 7, 2016.

BRASIL. Subsecretaria Nacional de Promoção dos Direitos da Pessoa com Deficiência. Comitê de Ajudas Técnicas. Tecnologia Assistiva. Brasília: CORDE, 138 p. 2009.

BRASIL. Portaria Interministerial No. 362 - Dispõe sobre o limite de renda mensal dos tomadores de recursos nas operações de crédito para aquisição de bens e serviços de Tecnologia Assistiva destinados às pessoas com deficiência e sobre o rol dos bens e serviços. 2012. Publicado em DOU No. 207, 24/10/2012. Disponível em: <http://www.pessoacomdeficiencia.gov.br/app/sites/default/files/arquivos/\%5Bfield_generico_imagens-filefield-description\%5D_58.pdf> Acesso em: 27 jul. 2017.

BUEHLER, E. et al. Investigating the Implications of 3D Printing in Special Educa- 
tion. ACM Transactions on Accessible Computing. v. 8, n. 3, p. 28, 2016.

BULGARELLI, A. A Low-cost Open Source 3D-Printable Dexterous Anthropomorphic Robotic Hand with a Parallel Spherical Joint Wrist for Sign Languages Reproduction. International Journal of Advanced Robotic Systems. v.13, n.126, p. 13, 2016.

COMOTTI, C.; et al. Additive Manufacturing to Advance Functional Design: An Application in the Medical Field. Journal of Computing and Information Science in Engineering. v.17, 2017.

DODZIUK, H. Applications of 3D printing in healthcare. Kardiochirurgia i Torakochirurgia Polska. v. 13, n.3, p. 283-293, 2016.

GRETSCH, K. F. et al. Development of novel 3D-printed robotic prosthetic for transradial amputees. Prosthetics and Orthotics International. v. 40, n. 3, p. 400403, 2015.

HANSEN, D. H.; OOSTHUIZEN, G. A.; GERHOLD, T. Resource-efficient process chains to manufacture patient-specific prosthetic fingers. South African Journal of Industrial Engineering. v. 27, n. 1, p. 75-87. 2016.

JUN, S. et al. Smart Knee Brace Design with Parallel Coupled Compliant Plate (PCCP) Mechanism and Pennate Elastic Band (PEB) Spring. Journal of Mechanisms and Robotics. v. 7, p. 13, 2015.

KAYE, R. et al. Three dimensional printing: A review on the utility within medicine and otolaryngology. International Journal of Pediatric Otorhinolaryngology. v. 89, p. 145-148, 2016.

LASZCZAK, P. et al. Development and validation of a 3D-printed interfacial stress sensor for prosthetic applications. Medical Engineering \& Physics. v. 37, p. 132-137, 2014.

MANTON, J. D. et al. Development of an open technology sensor suite for assisted living: a student-led research project. Interface Focus. v. 6, p. 13, 2016.

MARKSTEDT, K. et al. Bioprinting Human Chondrocytes with Nanocellulose -Alginate Bioink for Cartilage Tissue Engineering Applications. Biomacromolecules. v. 16, p. 1489-1496, 2015.

MARTELLI, N. et al. Advantages and disadvantages of 3-dimensional printing in surgery: A systematic review. Surgery. v. 159, n. 6, p. 1485-1500, 2016.

MARTIN, A. C. et al. Fabricación Digital Directa para Deportes y Aplicaciones Médicas: Tres Casos Prácticos. DYNA, v. 90, n. 6, p. 622-628, 2015. 
MICLAUS, R; REPANOVICI, A; ROMAN, N. Biomaterials: Polylactic Acid and 3D Printing Processes for Orthosis and Prosthesis. Revista de Materiale Plastice. v. 54, n.1, p. $98-102,2017$

ORTI, L. G.; CAZORLA, M. P.; MACIA, J. L. Improving Tactile Map Usability through 3D Printing Techniques: An Experiment with New Tactile Symbols. The Cartographic Journal. v. 52, n. 1. p. 51-57. 2015.

OSTUZZI, F. et al. + TUO project: low cost 3D printers as helpful tool for small communities with rheumatic diseases. Rapid Prototyping Journal. v. 21, n. 5, p. 491-505, 2015.

PALOUSEK, D. et al. Pilot study of the wrist orthosis design process. Rapid Prototyping Journal. v. 20, n. 1, p. 27-32, 2014.

RENGIER, F. et al. 3D printing based on imaging data: review of medical applications. International Journal of Computer Assisted Radiology and Surgery. v. 5, n. 4, p. 335-341, 2010.

RICO, Z. P. et al. Design of an exoskeleton for upper limb robot-assisted rehabilitation based on co-simulation. International Journal of Vibroengineering. v. 18, n. 5, p. 3269-3278, 2016.

TELFER, S. et al. Embracing additive manufacture: implications for foot and ankle orthosis design. BMC Musculoskeletal Disorders, v. 13, n. 84, p. 1471-1474, 2012.

TELFER. S. et al. Dose-response effects of customised foot orthoses on lower limb kinematics and kineticsin pronated foot type. Journal of Biomechanics. v. 46, p. 1489-1495, 2013.

TELFER. S. et al. Dose-response effects of customised foot orthoses on lower limb muscle activity and plantar pressures in pronated foot type. Gait \& Posture. v. 38, p. 443-449, 2013.

TZANG, M. J.; HSU, L. H.; SHANG, S. H. Development and Evaluation of a CAD/3DP Process for Transtibial Socket Fabrication. Biomedical Engineering: Applications, Basis and Communications. v. 27, n. 5, p. 9, 2015. 